\title{
Trivium
}

Revue franco-allemande de sciences humaines et sociales - Deutsch-französische Zeitschrift für Geistesund Sozialwissenschaften

15 | 2013

La science pense en plusieurs langues

\section{Plurilinguismus und Übersetzen: Investition in Europas Zukunft}

\section{Gerhard Stickel}

\section{OpenEdition}

Journals

Édition électronique

URL : http://journals.openedition.org/trivium/4557

DOI : $10.4000 /$ trivium.4557

ISSN : 1963-1820

\section{Éditeur}

Les éditions de la Maison des sciences de l'Homme

\section{Référence électronique}

Gerhard Stickel, «Plurilinguismus und Übersetzen: Investition in Europas Zukunft », Trivium [Online], 15 | 2013, online erschienen am 09 Dezember 2013, abgerufen am 08 September 2020. URL : http:// journals.openedition.org/trivium/4557 ; DOI : https://doi.org/10.4000/trivium.4557

Ce document a été généré automatiquement le 8 septembre 2020

\section{(c) (i) (9)}

Les contenus des la revue Trivium sont mis à disposition selon les termes de la Licence Creative Commons Attribution - Pas d'Utilisation Commerciale - Pas de Modification 4.0 International. 


\title{
Plurilinguismus und Übersetzen: Investition in Europas Zukunft
}

\author{
Gerhard Stickel
}

1 Unser Thema ist vermutlich nicht als rhetorische Frage gemeint. Als rhetorische Frage müsste es als unmittelbar plausibel suggerieren, dass Mehrsprachigkeit und Übersetzung kaum hinderlich und auch keine Vergeudung von Zeit und Geld seien, sie vielmehr beträchtlichen intellektuellen Mehrwert erzeugten. Das ist aber durchaus nicht selbstverständlich. Das Miteinander und Gegeneinander der vielen Sprachen in den Institutionen der Europäischen Union ist beschwerlich und verzögert die tagtägliche Arbeit. Die Beschäftigung von Tausenden von Übersetzern und Dolmetschern in Brüssel, Luxemburg und Straßburg kostet viel Geld. Das wird jedenfalls immer wieder beklagt: von einigen der politischen Akteure wie auch von manchen kritischen Beobachtern. Hinderlich ist die europäische Mehrsprachigkeit insgesamt auch für uns alle, weil sie uns die praktische Verständigung in Ländern erschwert, deren Sprache wir nur schlecht oder gar nicht können. Andererseits ist es in der sprachpolitischen Diskussion mittlerweile geradezu zum Topos geworden, dass der eigentliche Reichtum unseres Kontinents seine kulturelle und soziale Vielfalt ist und dass diese kulturelle Vielfalt Europas wesentlich auf der Vielfalt seiner Sprachen beruht.

2 Wir sind also zu einer Kosten-Nutzen-Rechnung auf gefordert, $\mathrm{zu}$ einer abwägenden Gegenüberstellung des Aufwands für Mehrsprachigkeit und Übersetzen einerseits und des damit erzielbaren Nutzens andererseits, der in der thematischen Frage als »intellektueller Mehrwert« bezeichnet wird. Zu bedenken ist dabei, dass der Titel sowohl in der deutschen wie der französischen Fassung mit seinen nominalen Ausdrücken weder Täter noch Betroffene nennt, also offen lässt, wer behindert wird, wer was vergeudet und wer welchen intellektuellen Mehrwert erhalten kann. Das ist zu klären. Zu berücksichtigen ist bei der Abwägung auch die Alternative, die uns oder unseren Nachfahren Mehrsprachigkeit und Übersetzen ersparen würde, nämlich Einsprachigkeit, Monolinguismus. 
3 Zunächst ein paar terminologische Festlegungen. Wenig Probleme sollte uns »Übersetzen« als Terminus bereiten. Der Einfachheit halber sollten wir für unsere Diskussion Dolmetschen unter Übersetzen mitverstehen. Bei »Mehrsprachigkeit« fehlt uns im Deutschen eine einfache Unterscheidungsmöglichkeit mit monoverbalen Ausdrücken, die in den romanischen Sprachen und im Englischen leichter fällt. $\mathrm{Zu}$ unterscheiden ist ja zwischen Mehrsprachigkeit als Fähigkeit eines einzelnen Menschen, in mehreren Sprachen kommunizieren zu können, und Mehrsprachigkeit als Eigenschaft von Gesellschaften und Bevölkerungen ganzer Länder oder Territorien, in denen mehr als eine Sprache gesprochen wird. Es geht um die Unterscheidung zwischen individueller Mehrsprachigkeit einerseits und sozialer oder territorialer Mehrsprachigkeit andererseits. Die beiden Arten der Mehrsprachigkeit treffen nur teilweise zusammen: In einer mehrsprachigen Gesellschaft oder einem mehrsprachigen Land sind nicht alle Menschen mehrsprachig. Nicht alle Belgier, Schweizer oder Finnen sind zwei- oder dreisprachig. In der neueren englischsprachigen Fachliteratur wird eine Unterscheidung gemacht mit den Termini plurilingualism für individuelle Mehrsprachigkeit und multilingualism für soziale und territoriale Mehrsprachigkeit. Im Französischen sollte eine analoge Unterscheidung zwischen plurilinguisme und multilingnisme ebenfalls leicht möglich sein. (Wie mir französische Kollegen gesagt haben, ist multilinguisme noch wenig gebräuchlich.) Auch im Deutschen können Ausdrücke wie Plurilinguismus und Multilinguismus gebildet werden, wenngleich sie etwas schwerfällig klingen.

Die verschiedenen Termini sind nötig, um die sprachliche Situation angemessen zu bezeichnen, in der wir in Europa leben. Sie ist ja in diesem Kolloquium schon einige Male angesprochen worden. Ich halte hierzu fest: Wenn auch die autochthonen Regional- und Minderheitensprachen berücksichtigt werden, gibt es im geographischen Europa je nach Zählung derzeit 70 bis 100 Sprachen. (Die Unschärfe resultiert vor allem aus Differenzen in der Unterscheidung zwischen Sprachen und Dialekten.) Im politischen Europa, der Europäischen Union, gibt es bekanntlich zur Zeit elf offizielle Sprachen der bisherigen 15 Mitgliedsländer. Es werden demnächst bis zu 21 offizielle Sprachen sein, wenn die zehn neuen Mitgliedsstaaten ihre Amtssprachen als zusätzliche Sprachen in die Institutionen der EU einbringen. Das hat Folgen für die Institutionen der Union und für die Mitgliedsländer.

5 Für die Institutionen sind die Folgen der Erweiterung akut und erheblich. Sie müssen das Miteinander, vielleicht auch Gegeneinander von 21 Sprachen im kommunikativen Alltag praktisch bewältigen. Ich möchte die verschiedenen Lösungsvorschläge, die alle auf eine Reduzierung der 21 offiziellen Sprachen auf eine bis hin $\mathrm{zu}$ sechs Arbeitssprachen hinauslaufen, nicht weiter erörtern. Ein Konsens ist zu dieser Frage bisher noch weniger erkennbar als zu den kritischen Punkten in dem vom Konvent vorgelegten Entwurf einer europäischen Verfassung. Soweit ich die Diskussion mitbekommen habe, zeichnet sich lediglich ab, dass mit zunehmender Anzahl der erwogenen bis zu sechs Arbeitssprachen der Widerstand der so genannten kleineren Staaten mit ihren Sprachen zunimmt und ihre Neigung zu möglichst nur einer einzigen Arbeitssprache. Warum sollten Dänen, Esten oder Slowenen sich mit bis zu sechs Arbeitssprachen abplagen, wenn ihre eigene Sprache für eine solche Funktion von vornherein nicht in Frage kommt. In jedem Fall wird der Aufwand an Übersetzungen zunächst weiter zunehmen. Plurilinguale Beamte in Brüssel oder Politiker, die mit 21 Sprachen umgehen können, gibt es meines Wissens nicht, Übersetzer wohl auch 
nicht. Erforderlich ist deshalb eine zunehmende Anzahl spezialisierter Übersetzer mit teilkomplementären Sprachkenntnissen.

Für die sprachlichen Verhältnisse in den derzeitigen und künftigen Mitgliedsstaaten hat die Erweiterung der Union zunächst nur wenig Konsequenzen. Die Deutschen können weiterhin untereinander deutsch reden, die Franzosen französisch und die Polen polnisch. Mit der engeren wirtschaftlichen Verflechtung und der höheren grenzüberschreitenden Mobilität der Europäer nehmen jedoch die Sprachkontakte zu, d. h. Begegnungen von Menschen unterschiedlicher Muttersprachcn, die jeweils zu ermitteln haben, ob sie sich in einer ihrer Sprachen oder einer dritten verständigen können oder ob sie Übersetzer benötigen. Dies gilt nicht nur für unmittelbare Gesprächsbegegnungen, sondern auch für alle anderen medialen Arten der Kommunikation, die auch personell ungerichtet sein kann, wenn es etwa darum geht, Zeitungen, Fernsehen oder die Literatur der anderen Länder zu verstehen.

7 Die multilingualen Verhältnisse in Europa insgesamt sind nur schwer gezielt $\mathrm{zu}$ beeinflussen. Die europäische Sprachenvielfalt ist historisch gewachsen. Sie ist zwar in Teilbereichen politisch veränderbar dadurch, dass etwa ein Staat den amtlichen Geltungsbereich einer seiner Sprachen ändert oder dass regionale Varietaten einer Sprache zu separaten Hochsprachen erklärt werden (Beispiel: Kroatisch und Serbisch). Sprachpolitische Maßnahmen zielen aber meist auf eine Verringerung der Vielfalt ab, indem Minderheitensprachen unterdrückt oder benachteiligt werden oder eine von mehreren Varietaten einer Sprache bevorzugt und zur überregionalen Standardsprache ausgebaut wird.

Plurilinguismus, also individuelle Mehrsprachigkeit, lässt sich anders als Multilinguismus gezielt fördern, vor allem durch entsprechende Erziehungsprogramme. Neben Kindern, die in multilingualen Familien aufwachsen und so gleichsam naturwüchsig plurilingual werden, haben viele Europäer monolingualer Herkunft zusätzlich zu ihrer Erstsprache eine oder gar mehr andere Sprachen hinzugelernt: in der Schule oder durch Aufenthalte in anderssprachiger Umgebung ${ }^{1}$. Inzwischen lernen die meisten Kinder in den europäischen Ländern in der Schule wenigstens eine Fremdsprache, werden also mehr oder weniger plurilingual.

9 Plurilinguismus und Übersetzung sind komplementäre Verfahren für den Umgang mit multilingualen Verhältnissen. Um in multilingualen Gesellschaften oder Territorien kommunizieren $\mathrm{zu}$ können, muss man entweder selbst plurilingual sein oder Übersetzer bemühen. Wer die Sprache seines Partners nicht kann, braucht Übersetzer. Ein plurilingualer Schweizer oder Belgier, der sich in den Sprachen seines Landes auskennt, braucht dagegen im eigenen Land keine Übersetzung. Wie steht es mit den Kosten?

Übersetzen ist teuer. Wenn auch Übersetzer durch ihre Arbeit nicht sonderlich reich werden können, ist eine Veranstaltung wie diese mit Simultanübersetzung in zwei Sprachen erheblich teurer als eine monolinguale Konferenz. Ich weiß nicht, ob schon jemand die ungefähren Kosten ermittelt hat, die in einem Land wie Deutschland oder Frankreich jahrlich für Übersetzungen aufgewendet werden: in der Wirtschaft, der Politik, den Wissenschaften, in den sprachbasierten Kulturbereichen von den Zeitungen über das Fernsehen bis hin zu Theater und Literatur. Insgesamt sind es wohl immense Summen, die für professionelle Dolmetscher und Übersetzer ausgegeben werden, von den Amateurübersetzern ganz zu schweigen. Zehntausende sind in ganz Europa tageintagaus mit Hin- und Herübersetzen zwischen europäischen und 
außereuropäischen Sprachen beschäftigt, selten im eigenen Interesse, meist für andere Menschen, denen die entsprechenden Sprachkenntnisse fehlen. Genaueres ist über die Kosten bekannt, welche die multilinguale Staatengemeinschaft den Institutionen der Europäischen Union bereitet, also dort, wo es zu besonders intensiven und vielfältigen Sprachkontakten kommt. Der dänische Linguist Robert Phillipson (2003) hat diese Kosten aus den offiziellen Dokumenten der EU zusammengestellt. 2001 kosteten demnach die Übersetzungsdienste aller europäischen Institutionen zusammen über 500 Mio Euro. Das sind zwar nur 0,8 \% der Haushaltsmittel der Union. ${ }^{2}$ Aber auch dieses Geld könnte für andere Zwecke ausgegeben werden, wenn sich die Mitgliedsstaaten auf eine einzige Arbeitssprache in den Institutionen der EU einigten. Damit würden zwar die Übersetzungsdienste nicht ganz entfallen, aber sie wären höchstens noch halb so teuer, weil die Anzahl der Sprachenpaare, zwischen denen noch zu übersetzen wäre, drastisch reduziert würde. ${ }^{3}$

$11 \mathrm{Zu}$ erwähnen sind in diesem Zusammenhang auch technische Übersetzungshilfen. Vor etwa zehn Jahren hatte ich in Paris ein längeres Gespräch mit Bernard Quemada, dem damaligen Vizepräsidenten des Conseil supérieur de la langue française. Wir erörterten die paradoxe Aufgabe, zum einen die praktischen Hindernisse des multilingualen Europas zu überwinden, zum anderen eben diese europäische sprachliche Vielfalt wegen ihrer kulturellen Bedeutung zu erhalten. Ich plädierte etwas hastig für vermehrten Fremdsprachenunterricht, den ich mir auch selbst gewünscht hatte, und besonders auch für das Erlernen rezeptiver Mehrsprachigkeit. Quemada war damais sehr optimistisch und riet zur Geduld. Wir brauchten nur noch etwa zehn bis $15 \mathrm{Jahre}$ $\mathrm{zu}$ warten, so meinte er; in dieser Zeit würden die praktischen Verständigungsprobleme weitgehend technisch gelöst werden. Die Verfahren der automatischen Sprachübersetzung würden bis dahin soweit verbessert sein, dass zwischen den europäischen Sprachen ohne weiteres mit Computern hin- und herübersetzt werden könne. Ich möchte nun nicht aus zehnjähriger Distanz den geschätzten Kollegen wegen seiner $\mathrm{zu}$ optimistischen Einschätzung des computerlinguistischen Fortschritts kritisieren. Wichtig ist mir lediglich, dass die automatische Sprachübersetzung, auch wenn sie perfektioniert werden kann, nur eine sehr begrenzte Teillösung wäre. Sie würde zur Verfestigung der in den meisten europäischen Landern noch vorherrschenden Einsprachigkeit beitragen, weil sie vielen Menschen das praktische Motiv für das mühsame Erlernen anderer Sprachen nähme. Andererseits könnte sie aber auch die sprachliche Vielfalt in Europa stutzen. Manchen Sprachen droht ja derzeit, dass sie nicht als »vollständige« Sprachen weiter bestehen können, weil ihre Sprecher in wichtigen kommunikativen Domänen aus ihrer Sprache in eine andere Sprache rauswandern`, zumeist in ein globalisiertes Englisch. Tendenziell trifft das selbst für relativ "große« Sprachen wie Französisch und Deutsch zu. Dies wurde in der Sektion 1 schon für die Sprachdomäne Wissenschaft erörtert. Neben den weiterhin benötigten menschlichen Übersetzern könnte die automatische Sprachübersetzung in Verbindung mit Terminologie-Datenbanken dazu beitragen, die gefährdete Fachkommunikation in den einzelnen Sprachen weiterhin zu ermöglichen. Aber auch die automatische Sprachübersetzung ist nicht kostenlos zu haben. Ich weiß nicht, wieviel Geld, Linguisten- und Programmiererfleiß die bisher noch unvollkommenen Systeme (Systran u. a.) schon gekostet haben. Ich vermute, es waren insgesamt beträchtliche Summen.

$\mathrm{Zu}$ den Kosten der individuellen Mehrsprachigkeit der Europäer kann ich keine Zahlen nennen. Es reicht ja nicht, die Kosten des Fremdsprachenunterrichts in Schulen und 
anderen Bildungseinrichtungen aller EU-Staaten $\mathrm{zu}$ addieren. Hinzu kommt der Aufwand an Zeit und Anstrengung, manchmal auch an Geld, den jeder Einzelne aufbringen muss, der zu seiner Erstsprache noch eine oder gar mehrere andere Sprachen hinzulernt, falls er die nicht in seiner multilingualen Familie oder auf der Straße gelernt hat. Aber auch dann ist der zusätzliche Spracherwerb nicht ohne die Kosten zusätzlicher Anstrengungen zu haben. Der Aufwand der Schulen zur Vermittlung individueller Mehrsprachigkeit wird immer wieder zum Argument in der deutschen Bildungspolitik, wenn diskutiert wird, inwieweit der Fremdsprachenunterricht zu Lasten des Unterrichts in anderen Fächern geht. Die Diskussion wird besonders lebhaft, wenn über die zweite Fremdsprache verhandelt wird. Statt vier Stunden Französisch oder Spanisch pro Woche könnten ja auch mehr Physik, Chemie oder Ökonomie unterrichtet werden, Fächer, die für das spätere Berufsleben vielleicht nützlicher sind als die Französisch- oder Spanischkenntnisse, die nach mehrjährigem Unterricht erreicht werden.

Die Fremdsprachenkenntnisse, die in der Schule oder sonstwo erworben werden, reichen ohnehin nicht aus, um alle Situationen des Sprachkontakts, die sich in Europa ergeben, $\mathrm{zu}$ bewältigen. Und dies nicht nur, weil die Schulkenntnisse für eine anspruchsvolle Kommunikation in der jeweiligen Zielsprache oft nicht ausreichen, sondern auch, weil es allein in der EU mehr Zielsprachen gibt, als ein einzelner bewältigen kann. Niemand kann zu seiner eigenen Sprache noch die zehn oder gar 20 anderen offiziellen Sprachen der EU alle lernen. Auch wenn das sog. Barcelona-Prinzip $M+2$ (Muttersprache plus zwei Fremdsprachen) ${ }^{4}$ in allen Ländern der EU verwirklicht werden kann, wird dies nicht $\mathrm{zu}$ einer homogenen Verteilung von Fremdsprachenkenntnissen in Europa führen. Sprachen wie Englisch, Französisch, Deutsch, neuerdings auch Spanisch, Würden und werden sicherlich häufiger in den Schulen unterrichtet und gelernt als etwa Tschechisch, Finnisch oder gar Maltesisch. Plurilinguismus allein wird also nicht genügen, um dem einzelnen Europäer alle Schranken des multilingualen Europas zu öffnen. Je nach seinen Kommunikations- und Mobilitätsbedürfnissen wird er auf Partner hoffen müssen, die seine Sprache können oder mit denen er sich in einer dritten Sprache verständigen kann, oder er wird die Hilfe von Übersetzern benötigen, für manche Zwecke vielleicht auch einen Übersetzungscomputer.

Wie steht es mit dem intellektuellen Gewinn von Mehrsprachigkeit und Übersetzung? Auch hier muss man trennen. Plurilinguismus, also der erfolgreiche Erwerb von Fremdsprachen, bringt zunächst einmal dem einzelnen Menschen Gewinn: Er gewinnt unmittelbaren Zugang zu einer anderen sprachgebundenen Kultur mit ihrer Geschichte und ihren aktuellen Ausprägungen. Er gewinnt damit zusätzliche sprachvermittelte Weltsichten. Er macht bei der Rezeption anderssprachiger Literatur ästhetische Erfahrungen, die ihm Übersetzungen allenfalls partiell vermitteln können. Auch wenn man diesen Nutzen als Lustgewinn weniger Intellektueller nicht sonderlich hoch bewerten möchte, ist der Gewinn an zusätzlichen kommunionspraktischen Handlungsräumen zu nennen, den die Fahigkeit bietet, mit Sprechern oder Texten der jeweils anderen Sprachen umzugehen, und zwar ohne Dolmetscher oder Übersetzer. Als Kaufmann, Diplomat oder Tourist kann ich mich in dem jeweiligen Sprachgebiet ohne fremde Hilfe bewegen. Eher in den Bereich des intellektuellen Lustgewinns gehört, dass der Umgang mit anderen Sprachen auch das Bewusstsein für die eigene Sprache und ihre Spezifika schärft und zu ihrer Bereicherung beiträgt. 

denken, den diese Tatigkeit dem Übersetzer bringt. Übersetzungen sind vor allem ein Gewinn für andere, für ihre Nutzer, für die Menschen, die ohne die Leistungen der Übersetzer keinen Zugang zu Texten und Äußerungen in der jeweils anderen Sprache hätten. Da der Erwerb von Fremdsprachenkenntnissen für die meisten Menschen sehr begrenzt ist, wird ihnen die Erfahrung der meisten anderssprachigen Kulturen wie in der Vergangenheit auch künftig nur über Übersetzungen möglich sein. Wichtige Züge der europäischen Geistesgeschichte lassen sich ja als Übersetzungsgeschichte zeichnen: von der Übertragung griechischer und lateinischer Texte in die verschiedenen Volkssprachen bis zu den wechselseitigen Übersetzungen aus den Sprachen, die sich seit dem Mittelalter nach und nach vom Lateinischen emanzipiert haben. Angesichts der heute noch gegebenen Sprachenvielfalt in Europa wird auch künftig auf Übersetzungen nicht zu verzichten sein.

sei denn, diese sprachliche Vielfalt Europas würde aufgegeben. Nicht schlagartig natürlich, sondern nach und nach, so dass für einige Zeit noch Bedarf an Fremdsprachenkenntnissen und Übersetzern bliebe, der aber in dem Maße zurückginge, in dem alle Europäer sich für eine und dieselbe Sprache entschlössen und nach ein, zwei Generationen über einen entsprechenden Sprachunterricht in den Schulen schließlich alle Europäer einsprachig würden. Fremdsprachenkenntnisse und Übersetzer wären dann allenfalls noch für den Außenverkehr erforderlich, mit Menschen in außereuropäischen Ländern, die noch nicht die in Europa geltende Einheitssprache gelernt hätten. Die skizzierten Kosten an Zeit, Mühe und Geld könnten weitgehend entfallen. Europa würde damit freilich nach und nach seine kulturelle Vielfalt aufgeben, die heute noch von vielen als sein eigentlicher Reichtum angesehen wird.

\section{Unsicherheitsfaktoren bleiben. Zusammenfassend lässt sich sagen:}

1. Die Kosten von Plurilinguismus und Übersetzen sind hoch. Das Erlernen anderer Sprachen bleibt mühsam und zeitraubend. Der Aufwand für Übersetzungsdienste in den einzelnen Staaten und in den Institutionen der EU wird kaum zurückgehen, auch wenn mehr und mehr Akteure plurilingual sein werden. Von den Kosten der Übersetzungsdienste sollten wir uns dennoch nicht zu sehr beeindrucken lassen. 0,8\% des EU-Haushalts bedeutet, dass für alle Übersetzungsdienste der EU auf jeden Bürger der Union jährlich 2 Euro entfallen. Ebensoviel entfallen auf den Einzelnen auch für die Subvention der Milchkühe in den EU-Ländern.

19 2. Plurilinguismus ist wertvoll und nützlich für den einzelnen Deutschen, Franzosen oder sonstigen Europäer, der mit der Kenntnis anderer Sprachen einen direkten Zugang $\mathrm{zu}$ anderssprachigen Kulturen hat und mit anderssprachigen Europäern leichter kommunizieren kann. Und: Wer andere Sprachen lernt, tut nicht nur etwas für diese Sprachen, sondern auch für seine eigene. Übersetzungen waren schon immer wichtig für den kulturellen und wirtschaftlichen Austausch zwischen den verschiedenen Sprachgemeinschaften in Europa. Sie sind weiterhin nötig. Sie sind eine unabdingbare Ergänzung zu den stets beschränkten Fremdsprachkenntnissen der einzelnen Menschen. Plurilinguismus und Übersetzungen sind erforderlich zur Wahrung der europäischen Sprachenvielfalt und ihrer Weiterentwicklung. Die Kosten hierfür sollten deshalb weniger als vermeidbare Ausgaben denn als Investitionen in die Zukunft Europas gesehen werden. 
20 3. Nicht ganz klar ist mir, ob nicht auch konsequente Zweisprachigkeit (Bilinguismus) sowohl den Sprachverkehr zwischen den verschiedensprachigen Europäern erleichtern wie auch zur Erhaltung der sprachlichen Vielfalt beitragen könnte. Bilinguismus hieße, dass alle Europäer neben ihrer jeweiligen Muttersprache (und eventuellen weiteren offiziellen Sprachen ihres Landes) nur noch eine und dieselbe Fremdsprache als gemeinsame Verkehrsprache zu lernen hätten. Dies würde den Aufwand für Übersetzungen und für den Erwerb von Fremdsprachenkenntnissen erheblich reduzieren. Die Zugänge zu anderssprachigen Kulturen und auch das Vergnügen daran würden so freilich erschwert, weil sie stets über die Einheitssprache zu vermitteln wären. Auch die Kommunikation in den eigenen Sprachen europäischer Partner aus verschiedenen Ländern entfiele weitgehend. Tendenziell haben wir derzeit eine solche Entwicklung mit der Bevorzugung von Englisch als erster Fremdsprache in den meisten Staaten der Union. Ich vermute, dass eine solche Entwicklung den einzelsprachlichen Kulturen und der sprachlichen Vielfalt Europas insgesamt letztlich wenig nützen, vielleicht sogar schaden würde. Denn auf diese Weise könnte sich eine Diglossie zum Nachteil der Einzelsprachen entwickeln, d.h. eine Trennung der sprachlichen Domänen: Wichtige Angelegenheiten in Politik, Wissenschaft und Wirtschaft würden zunehmend nur noch in der europäischen Einheitssprache verhandelt und der Gebrauch der heimischen Sprachen würde mehr und mehr eingeschränkt auf Familie, Freizeit und Folklore.

21 Ich kann jedoch nicht einschätzen, wie wahrscheinlich eine solche Entwicklung ist, wenn nicht gegengesteuert wird. Möglicherweise kennen Sie, meine Damen und Herren, Symptome und Argumente, die zu einem deutlicheren Ergebnis der Abwägung der Vor- und Nachteile von Mehrsprachigkeit und Übersetzen führen, die zudem meine Vermutung erhärten oder auch widerlegen, dass konsequente Zweisprachigkeit aller Europäer zur Wahrung der sprachlichen Vielfalt unseres Kontinents nicht ausreicht.

\section{NOTES}

1. Nach einer Umfrage des Eurobarometers vom Frühjahr 2001 sollen 47 \% der Bürger der EUStaaten in der Lage sein, sich an einem Gespräch in einer anderen Sprache als ihrer Muttersprache zu beteiligen. Es gibt aber große Unterschiede zwischen den Staaten. Während fast alle Luxemburger (97\%) sich in wenigstens einer zweiten Sprache unterhalten können, ist weniger als ein Drittel der Briten (27 \%) dazu fähig. Deutsche (53 \%) und Franzosen (47 \%) liegen mit ihren Fremdsprachenkenntnissen nach dieser Umfrage im Mittelfeld. Hierzu: Europäische Kommission, Eurobarometer - Die öffentliche Meinung in Europa. Bericht Nr. 55, Ausgabe Oktober 2001. (Internet: http://europa.eu.int/comm/public_opinion).

2. Auf jeden Bürger der Union entfallen damit rund 2 Euro pro Jahr für die Übersetzerdienste der EU. Siehe hierzu Phillipson, Robert, 2003: English-Only Europe? Challenging Language Policy. London/New York: Routledge, S. 114.

3. Für die derzeit 11 Amts- und Arbeitssprachen der EU sind es für Übersetzungen insgesamt 110 Sprachenpaare (beide Richtungen gezählt). Wäre eine der elf Sprachen gemeinsame 
Arbeitsprache, wären es nur noch 20 Paare bzw. Übersetzungsrichtungen. Mit einer Arbeitssprache aus 21 Sprachen wären es 40 Übersetzungspaare.

4. Benannt nach einer von den Bildungsministern der EU-Staaten am 31.3.95 in Barcelona gefassten Entschließung. Siehe: Europäische Kommissionm Weißbuch zur allgemeinen und beruflichen Bildung. Luxemburgm Amt für amtliche Veröffentlichungen der europäischen Gemeinschaftm 1996, S. 72-74.

INDEX

Mots-clés : plurilinguisme, diversité linguistique, traduction

Schlüsselwörter : Plurilinguismus, Sprachenvielfalt, Übersetzung

\section{AUTEUR}

\section{GERHARD STICKEL}

Gerhard Stickel ist Honorarprofessor für Linguistik an der Universität Mannheim. Nähere Informationen finden Sie hier. 